\title{
Rotationally invariant singular solutions to the Kapustin-Witten equations
}

SIQI HE

In the present paper, we find a system of non-linear ODEs that gives rotationally invariant solutions to the Kapustin-Witten equations in 4-dimensional Euclidean space. We explicitly solve these ODEs in some special cases and find decaying rational solutions, which provide solutions to the Kapustin-Witten equations. The imaginary parts of the solutions are singular. By rescaling, we find some limit behavior for these singular solutions. In addition, for any integer $k$, we can construct a $5|k|$ dimensional family of $C^{1}$ solutions to the Kapustin-Witten equations on Euclidean space, again with singular imaginary parts. Moreover, we get solutions to the Kapustin-Witten equation with Nahm pole boundary condition over $S^{3} \times(0,+\infty)$.

4 Instanton number zero solutions

5 Non-zero instanton number solutions

$6 \quad$ Nahm pole boundary solution over $S^{3} \times(0,+\infty)$ 


\section{Introduction}

In [13, Witten proposed a new physical interpretation of the Jones polynomial and Khovanov homology in terms of counting the solutions of a certain supersymmetric gauge theory in four dimensions and five dimensions. The BPS equations of $\mathrm{N}=4$ twisted super Yang-Mills theory in four dimensions are called the topological twisted equations [6] and play an essential role in this framework.

Let $X$ be a connected, smooth, oriented 4-manifold with a Riemannian metric. Let $P$ be a chosen principle $S U(2)$ bundle over $X$ and let $\operatorname{ad}(P)$ denote the adjoint bundle of $P$. The topological twisted equations are equations for a pair $(A, \phi)$ where $\mathrm{A}$ is a connection on $P$ and $\phi$ is a $\operatorname{ad}(P)$ valued 1 -form. These equations have the following form

$$
\begin{aligned}
\left(F_{A}-\phi \wedge \phi+\lambda d_{A} \phi\right)^{+} & =0, \\
\left(F_{A}-\phi \wedge \phi-\lambda^{-1} d_{A} \phi\right)^{-} & =0, \\
d_{A}^{\star} \phi & =0 .
\end{aligned}
$$

Witten points out that the most interesting case to study is when $\lambda=-1$. In this case, we obtain the following equations, which we call the KapustinWitten equations:

$$
\begin{aligned}
F_{A}-\phi \wedge \phi-\star d_{A} \phi & =0, \\
d_{A}^{\star} \phi & =0 .
\end{aligned}
$$

In [6], Kapustin and Witten prove that over a closed manifold, all the regular solutions to the Kapustin-Witten equations are flat $S L(2 ; \mathbb{C})$ connections. Therefore, the regular solutions to these equations are not so interesting over closed manifolds. However, the Kapustin-Witten equations are interesting over non-compact spaces with singular boundary conditions. Witten's gauge theory approach [13] to the Jones polynomial conjectures that the coefficients of the Jones polynomial of a knot are determined by counting the solutions to the Kapustin-Witten equations with Nahm pole boundary conditions. See also Gaiotto and Witten [5] for an approach to this conjecture. The case of the empty knot is resolved in [7].

In addition, Taubes studied the compactness properties of the KapustinWitten equations [10] 9]. He shows that there can be only two sources of non-compactness. One is the traditional Uhlenbeck bubbling phenomenon [11 [12, and another is the non-compactness coming from the unboundness of the $L^{2}$ norm of $\phi$. 
Therefore, a natural question to ask is whether the Uhlenbeck bubbling phenomenon can appear for solutions to the Kapustin-Witten equations. In addition, do we have a model solution to the Kapustin-Witten equations.

In this paper, we construct some singular solutions to the KapustinWitten equations.

To be more precise, consider the trivial $S U(2)$ bundle $P_{0}$ over $\mathbb{R}^{4}$. Denote $x$ to be a point in $\mathbb{R}^{4}$, after identifying $\mathbb{R}^{4}$ with the quaternions $\mathbb{H}$ and the adjoint bundle $\operatorname{ad}\left(P_{0}\right)$ with imaginary part of the quaternions, we prove the following:

Theorem 1.1. For any real number $C$, the formulas

$$
\left\{\begin{array}{l}
A(x)=\operatorname{Im}\left(\frac{3 C}{C^{2}|x|^{4}+4 C|x|^{2}+1} \bar{x} d x\right) \\
\phi(x)=\operatorname{Im}\left(\frac{3 C\left(C|x|^{2}+1\right)}{\left(C^{2}|x|^{4}+4 C|x|^{2}+1\right)\left(C|x|^{2}-1\right)} \bar{x} d x\right)
\end{array}\right.
$$

give solutions to the Kapustin-Witten equations (2) with the following properties:

(1) For $C \neq 0$, the solutions are smooth away from $|x|=\frac{1}{\sqrt{C}}$ and decay to 0 when $|x| \rightarrow \infty$.

(2) The solutions have instanton number 0 .

(3) When $C \rightarrow+\infty,\left|F_{A}\right|$ converges to a Dirac measure at $x=0$.

(4) For $C \neq 0$, the pole singularity of $\phi$ at $|x|=\frac{1}{\sqrt{C}}$ cannot be removed by $S U(2)$ gauge transformations.

In addition, we also prove the following theorem:

Theorem 1.2. There exists a family of rotationally invariant solutions to the Kapustin-Witten equations on Euclidean $\mathbb{R}^{4}$ with instanton number \pm 1 . These solutions are smooth away from a sphere where the real parts are $C^{1}$ and the imaginary parts are singular.

In addition, given an integer $k$, we can generalize the ADHM construction [2] and obtain the following theorem:

Theorem 1.3. Given an integer $k$, there exists a $5|k|$ dimensional family of singular solutions to the Kapustin-Witten equations on Euclidean $\mathbb{R}^{4}$. When $k= \pm 1$, these include the solutions from Theorem 1.2.

We conjecture that under some non-degeneracy condition, the solutions we obtain in Theorem 1.3 have instanton number $k$. 
In addition, Witten in [13] suggested to study solutions to (2) with a singular boundary condition called the Nahm pole boundary condition. Solutions with the Nahm pole boundary condition play an important role in the gauge theory approach to the Jones polynomial.

In the last chapter, we observe the relation between the singularity which appears in Thm 1.1 and the Nahm pole boundary condition and get the following theorem:

Theorem 1.4. There exist two Nahm pole solutions to the Kapustin-Witten on $S^{3} \times(0,+\infty)$, with instanton number $\frac{1}{2}$ and $-\frac{1}{2}$.

Outline. In Section 2, we find a system of non-linear ODEs which will give rotationally invariant solutions to the Kapustin-Witten equations. In Section 3, we find a first integral of these ODEs and solve them to obtain the solutions in Theorem 1.1. In Section 4, we prove the rest part of Theorem 1.1. In Section 5, we construct other families of solutions to the KapustinWitten equations and prove Theorem 1.2 and Theorem 1.3. In Section 6, we build up the relation of our singular solution and Nahm pole.

\section{ODEs from the Kapustin-Witten equations}

\subsection{Background}

In accordance with the philosophy of the ADHM construction [1] [2] for the anti-self-dual equation, we use quaternions to describe the gauge field in $\mathbb{R}^{4}$. We begin by briefly recalling the elementary properties of quaternions.

We have three elements $I, J, K$ satisfying the identities: $I^{2}=J^{2}=$ $K^{2}=-1, I J=-J I=K, J K=-K J=I, K I=-I K=J$. A general quaternion $x$ is of the following form:

$$
x=x_{1}+x_{2} I+x_{3} J+x_{4} K
$$

where $x_{1}, x_{2}, x_{3}, x_{4}$ are real numbers. After choosing a canonical basis of $\mathbb{R}^{4}$, we can naturally identify points in $\mathbb{R}^{4}$ with quaternions. The conjugate quaternion is given by

$$
\bar{x}=x_{1}-x_{2} I-x_{3} J-x_{4} K
$$

and with we have the relation $\overline{x y}=\bar{y} \bar{x}$. In addition, we also know that $x \bar{x}=\bar{x} x=|x|^{2}=\sum x_{i}^{2}$. For $x=x_{1}+x_{2} I+x_{3} J+x_{4} K$, the imaginary part of $\mathrm{x}$ is $\operatorname{Im}(x):=x_{2} I+x_{3} J+x_{4} K$. Therefore, the Lie group $S U(2)$ can be 
identified with the unitary quaternions and the Lie algebra $\mathbf{s u}(2)$ can be identified with the imaginary part of the quaternions.

Using the well known isomorphism of the Lie group $S O(4)$ with $S U(2) \times$ $S U(2) / \sim$, the action of $S O(4)$ on a quaternion $x$ is given by $x \rightarrow a x b$, where $a, b$ are unitary quaternions.

In order to find rotationally invariant solutions, we assume that the gauge fields of (1) (2) have the following form:

$$
\begin{aligned}
A(x) & :=\operatorname{Im}(f(t) \bar{x} d x) \\
\phi(x) & :=\operatorname{Im}(g(t) \bar{x} d x) \\
t & :=|x|^{2} .
\end{aligned}
$$

Here $f(t), g(t)$ are real functions with variable $t=|x|^{2}$. Obviously, $t \geq 0$.

Remark. In the remaining part of the paper, we use $f^{\prime}, g^{\prime}$ to simplify writing $\frac{d f(t)}{d t}$ and $\frac{d g(t)}{d t}$.

Proposition 2.1. $A(x)$ and $\phi(x)$ defined as in (4) are rotationally invariant up to gauge equivalence.

Proof. It is easy to see that for $a, b$ are two unitary quaternions, under the change $x \rightarrow a x b$, we obtain $|a x b|^{2}=|x|^{2}, A(a x b)=\operatorname{Im}(f(t) \overline{a x b} d(a x b))=$ $\bar{b} \operatorname{Im}(f(t) \bar{x} d x) b$. Therefore, $A(a x b)$ is gauge equivalent to $A(x)$ by a constant gauge transformation. Similarly, we can show $\phi(x)$ is also rotationally invariant up to the same gauge transformation.

\subsection{Basic properties of rotationally invariant connections}

As the equations (2) depend on the metric, we need to be explicit about the metric we choose.

Definition 2.2. A metric $g$ on $\mathbb{R}^{4}$ is called rotationally invariant if in quaternion coordinate $g=h(t) d x \otimes d \bar{x}$. $h(t)$ here is a positive function, $t=$ $|x|^{2}$.

Example 2.3. The Euclidean metric $d x \otimes d \bar{x}$ and the round metric $\frac{4}{(1+t)^{2}} d x$ $\otimes d \bar{x}$ on $\mathbb{R}^{4}$ are both rotationally invariant metrics.

Remark. In the rest of the paper, all the metrics we considered are rotationally invariant. 
Now, we will introduce some basic properties of connections in (4).

Lemma 2.4. $\operatorname{Im}(\bar{x} d x \wedge \bar{x} d x)=-\frac{1}{2}|x|^{2} d \bar{x} \wedge d x-\frac{1}{2} \bar{x} d x \wedge d \bar{x} x$.

Proof. Since the wedge product of a real form with itself is zero, we know that

$$
\Re(\bar{x} d x) \wedge \Re(\bar{x} d x)=0 .
$$

Since

$$
\Re(\bar{x} d x)=\frac{\bar{x} d x+d \bar{x} x}{2},
$$

we obtain

$$
\begin{aligned}
0 & =\Re(\bar{x} d x) \wedge \Re(\bar{x} d x) \\
& =\frac{(\bar{x} d x+d \bar{x} x) \wedge(\bar{x} d x+d \bar{x} x)}{4} \\
& =\frac{\bar{x} d x \wedge \bar{x} d x+\bar{x} d x \wedge d \bar{x} x+t d \bar{x} \wedge d x+d \bar{x} x \wedge d \bar{x} x}{4} .
\end{aligned}
$$

In addition, we have

$$
\operatorname{Im}(\bar{x} d x \wedge \bar{x} d x)=\frac{\bar{x} d x \wedge \bar{x} d x+d \bar{x} x \wedge d \bar{x} x}{2} .
$$

The plus sign on the right hand side of the above identity is because given two quaternion one forms $\omega_{1}, \omega_{2}$, we have $\overline{\omega_{1} \wedge \omega_{2}}=-\bar{\omega}_{2} \wedge \bar{\omega}_{1}$.

The result follows immediately.

Lemma 2.5. $\operatorname{Im}(\bar{x} d x) \wedge \operatorname{Im}(\bar{x} d x)=\operatorname{Im}(\bar{x} d x \wedge \bar{x} d x)$.

Proof. We calculate that

$$
\begin{aligned}
& \operatorname{Im}(\bar{x} d x) \wedge \operatorname{Im}(\bar{x} d x) \\
= & \frac{(\bar{x} d x-d \bar{x} x) \wedge(\bar{x} d x-d \bar{x} x)}{4} \\
= & \frac{\bar{x} d x \wedge \bar{x} d x-\bar{x} d x \wedge d \bar{x} x-t d \bar{x} \wedge d x+d \bar{x} x \wedge d \bar{x} x}{4} \\
= & \frac{\bar{x} d x \wedge \bar{x} d x+d \bar{x} x \wedge d \bar{x} x}{2}(\text { by Lemma 2.4) } \\
= & \operatorname{Im}(\bar{x} d x \wedge \bar{x} d x)
\end{aligned}
$$




\section{Lemma 2.6.}

$$
\begin{aligned}
d x \wedge d \bar{x}= & -2\left(\left(d x_{1} \wedge d x_{2}+d x_{3} \wedge d x_{4}\right) I+\left(d x_{1} \wedge d x_{3}+d x_{4} \wedge d x_{2}\right) J\right. \\
& \left.+\left(d x_{1} \wedge d x_{4}+d x_{2} \wedge d x_{3}\right) K\right) \\
d \bar{x} \wedge d x= & 2\left(\left(d x_{1} \wedge d x_{2}+d x_{4} \wedge d x_{3}\right) I+\left(d x_{1} \wedge d x_{3}+d x_{2} \wedge d x_{4}\right) J\right. \\
& \left.+\left(d x_{1} \wedge d x_{4}+d x_{3} \wedge d x_{2}\right) K\right) .
\end{aligned}
$$

Proof. By direct computation.

Remark. Given a rotationally invariant metric $g$ (Definition 2.2), we can define the Hodge star operator with respect to $g$. Denote $\Omega^{2+}\left(\Omega^{2-}\right)$ to be the self-dual (anti-self-dual) two-forms with respect to the Hodge star operator. It is easy to see that $d x \wedge d \bar{x} \in \Omega^{2+}$ and $d \bar{x} \wedge d x \in \Omega^{2-}$. Also $\operatorname{Im}(d x \wedge d \bar{x})=$ $d x \wedge d \bar{x}$ and $\operatorname{Im}(d \bar{x} \wedge d x)=d \bar{x} \wedge d x$.

\subsection{Separating terms in the topological-twisted equations}

Since the equations in (1) are separated into the self-dual parts and the antiself-dual parts, we also want to separate our calculation into the self-dual parts and the anti-self-dual parts.

Lemma 2.7. For $A(x)$ defined as in (4), we have

$$
\begin{aligned}
& F_{A}^{+}=-\frac{1}{2}\left(f^{\prime}+f^{2}\right) \bar{x} d x \wedge d \bar{x} x \\
& F_{A}^{-}=\left(\frac{1}{2} t f^{\prime}-\frac{1}{2} t f^{2}+f\right) d \bar{x} \wedge d x
\end{aligned}
$$

Proof. We calculate that

$$
\begin{aligned}
F_{A}= & d A+A \wedge A \\
= & d \operatorname{Im}(f \bar{x} d x)+\operatorname{Im}\left(f^{2} \bar{x} d x \wedge \bar{x} d x\right) \\
= & \operatorname{Im}(d f \bar{x} d x)+\operatorname{Im}(f d \bar{x} \wedge d x)+\operatorname{Im}\left(f^{2} \bar{x} d x \wedge \bar{x} d x\right) \\
= & \operatorname{Im}\left(\left(f^{\prime}+f^{2}\right) \bar{x} d x \wedge \bar{x} d x\right)+\operatorname{Im}\left(\left(f^{\prime} t+f\right) d \bar{x} \wedge d x\right)\left(\text { by } x \bar{x}=|x|^{2}=t\right) \\
= & -\frac{1}{2}\left(f^{\prime}+f^{2}\right) t d \bar{x} \wedge d x+\left(f^{\prime} t+f\right) d \bar{x} \wedge d x-\frac{1}{2}\left(f^{\prime}+f^{2}\right) \bar{x} d x \wedge d \bar{x} x \\
& (\text { by Lemma 2.4 } \\
= & \left(\frac{1}{2} t f^{\prime}-\frac{1}{2} t f^{2}+f\right) d \bar{x} \wedge d x-\frac{1}{2}\left(f^{\prime}+f^{2}\right) \bar{x} d x \wedge d \bar{x} x .
\end{aligned}
$$

The result follows immediately. 
Lemma 2.8. For $\phi(x)$ defined as in (4), we have

$$
\begin{aligned}
& (\phi \wedge \phi)^{+}=-\frac{1}{2} g^{2} \bar{x} d x \wedge d \bar{x} x \\
& (\phi \wedge \phi)^{-}=-\frac{1}{2} g^{2} t d \bar{x} \wedge d x
\end{aligned}
$$

Proof. We calculate that

$$
\begin{aligned}
\phi \wedge \phi & =\operatorname{Im}(g \bar{x} d x) \wedge \operatorname{Im}(g \bar{x} d x) \\
& =\operatorname{Im}\left(g^{2} \bar{x} d x \wedge \bar{x} d x\right)(\text { by Lemma 2.5) } \\
& =-\frac{1}{2} g^{2} t d \bar{x} \wedge d x-\frac{1}{2} g^{2} \bar{x} d x \wedge d \bar{x} x .(\text { by Lemma 2.4 }
\end{aligned}
$$

Lemma 2.9. For $(A(x), \phi(x))$ defined as in (4), we have

$$
\begin{aligned}
& \left(d_{A} \phi\right)^{+}=-\frac{1}{2}\left(g^{\prime}+2 f g\right) \bar{x} d x \wedge d \bar{x} x \\
& \left(d_{A} \phi\right)^{-}=\left(\frac{1}{2} g^{\prime} t+g-f g t\right) d \bar{x} \wedge d x
\end{aligned}
$$

Proof. We calculate that

$$
\begin{aligned}
d_{A} \phi & =d \phi+A \wedge \phi+\phi \wedge A \\
& =d \operatorname{Im}(g \bar{x} d x)+\operatorname{Im}(2 f g \bar{x} d x \wedge \bar{x} d x)(\text { by Lemma 2.5) } \\
& =\operatorname{Im}(d g \bar{x} d x)+\operatorname{Im}(g d \bar{x} \wedge d x)+\operatorname{Im}(2 f g \bar{x} d x \wedge \bar{x} d x) \\
& =\operatorname{Im}\left(\left(g^{\prime}+2 f g\right) \bar{x} d x \wedge \bar{x} d x\right)+\operatorname{Im}\left(\left(t g^{\prime}+g\right) d \bar{x} \wedge d x\right) \\
& =\left(-\frac{1}{2}\left(g^{\prime}+2 f g\right) t+\left(g^{\prime} t+g\right)\right) d \bar{x} \wedge d x-\frac{1}{2}\left(g^{\prime}+2 f g\right) \bar{x} d x \wedge d \bar{x} x \\
& =\left(\frac{1}{2} g^{\prime} t+g-f g t\right) d \bar{x} \wedge d x-\frac{1}{2}\left(g^{\prime}+2 f g\right) \bar{x} d x \wedge d \bar{x} x .
\end{aligned}
$$

Now, we will discuss the third equation of (1).

At first, we have the following identity:

Lemma 2.10. $x_{1} \operatorname{Im}(\bar{x})+x_{2} \operatorname{Im}(\bar{x} I)+x_{3} \operatorname{Im}(\bar{x} J)+x_{4} \operatorname{Im}(\bar{x} K)=0$. 
Proof. We calculate that

$$
\begin{aligned}
& x_{1} \operatorname{Im}(\bar{x})+x_{2} \operatorname{Im}(\bar{x} I)+x_{3} \operatorname{Im}(\bar{x} J)+x_{4} \operatorname{Im}(\bar{x} K) \\
= & x_{1}\left(-x_{2} I-x_{3} J-x_{4} K\right)+x_{2}\left(x_{1} I+x_{3} K-x_{4} J\right) \\
& +x_{3}\left(x_{1} J-x_{2} K+x_{4} I\right)+x_{4}\left(x_{1} K+x_{2} J-x_{3} I\right) \\
= & 0 .
\end{aligned}
$$

Lemma 2.11. Given a rotational invariant metric $h(t) d x \otimes d \bar{x}$, denote $\star$ to be the Hodge star operator with respect to this metric, we have $d(\operatorname{Im}(\bar{x})$ * $\left.d x_{1}+\operatorname{Im}(\bar{x} I) \star d x_{2}+\operatorname{Im}(\bar{x} J) \star d x_{3}+\operatorname{Im}(\bar{x} K) \star d x_{4}\right)=0$.

Proof. By definition, we have

$$
\operatorname{Im}(\bar{x})=-x_{2} I-x_{3} J-x_{4} K, \quad \star\left(d x_{1}\right)=h d x_{2} \wedge d x_{3} \wedge d x_{4},
$$

therefore $(d \operatorname{Im}(\bar{x})) \star d x_{1}=0$. Similarly, we have

$$
(d \operatorname{Im}(\bar{x} I)) \star d x_{2}=(d \operatorname{Im}(\bar{x} J)) \star d x_{3}=(d \operatorname{Im}(\bar{x} K)) \star d x_{4}=0 .
$$

Therefore,

$$
\begin{aligned}
& d\left(\operatorname{Im}(\bar{x}) \star d x_{1}+\operatorname{Im}(\bar{x} I) \star d x_{2}+\operatorname{Im}(\bar{x} J) \star d x_{3}+\operatorname{Im}(\bar{x} K) \star d x_{4}\right) \\
= & 2 h^{\prime}\left(x_{1} \operatorname{Im}(\bar{x})+x_{2} \operatorname{Im}(\bar{x} I)+x_{3} \operatorname{Im}(\bar{x} J)+x_{4} \operatorname{Im}(\bar{x} K)\right) \\
= & 0 .
\end{aligned}
$$

Lemma 2.12. For $(A(x), \phi(x))$ defined as in (4), we have

$$
A \wedge \star \phi+\star \phi \wedge A=0 .
$$

Proof. For $A \wedge \star \phi$, we calculate that

$$
\begin{aligned}
& A \wedge \star \phi \\
= & f g \operatorname{Im}(\bar{x} d x) \wedge \star \operatorname{Im}(\bar{x} d x) \\
= & f g\left(\operatorname{Im}(\bar{x}) d x_{1}+\operatorname{Im}(\bar{x} I) d x_{2}+\operatorname{Im}(\bar{x} J) d x_{3}+\operatorname{Im}(\bar{x} K) d x_{4}\right) \\
& \wedge\left(\operatorname{Im}(\bar{x}) \star d x_{1}+\operatorname{Im}(\bar{x} I) \star d x_{2}+\operatorname{Im}(\bar{x} J) \star d x_{3}+\operatorname{Im}(\bar{x} K) \star d x_{4}\right) \\
= & f g\left(\operatorname{Im}(\bar{x}) \operatorname{Im}(\bar{x}) d x_{1} \wedge \star d x_{1}+\operatorname{Im}(\bar{x} I) \operatorname{Im}(\bar{x} I) d x_{2} \wedge \star d x_{2}\right. \\
& \left.+\operatorname{Im}(\bar{x} J) \operatorname{Im}(\bar{x} J) d x_{3} \wedge \star d x_{3}+\operatorname{Im}(\bar{x} K) \operatorname{Im}(\bar{x} K) d x_{4} \wedge \star d x_{4}\right) .
\end{aligned}
$$


In addition, we calculate that

$$
\begin{aligned}
& \star \phi \wedge A \\
= & f g \star \operatorname{Im}(\bar{x} d x) \wedge \operatorname{Im}(\bar{x} d x) \\
= & f g\left(\operatorname{Im}(\bar{x}) \star d x_{1}+\operatorname{Im}(\bar{x} I) \star d x_{2}+\operatorname{Im}(\bar{x} J) \star d x_{3}+\operatorname{Im}(\bar{x} K) \star d x_{4}\right) \\
& \wedge\left(\operatorname{Im}(\bar{x}) d x_{1}+\operatorname{Im}(\bar{x} I) d x_{2}+\operatorname{Im}(\bar{x} J) d x_{3}+\operatorname{Im}(\bar{x} K) d x_{4}\right) \\
= & f g\left(\operatorname{Im}(\bar{x}) \operatorname{Im}(\bar{x}) \star d x_{1} \wedge d x_{1}+\operatorname{Im}(\bar{x} I) \operatorname{Im}(\bar{x} I) \star d x_{2} \wedge d x_{2}\right. \\
& \left.+\operatorname{Im}(\bar{x} J) \operatorname{Im}(\bar{x} J) \star d x_{3} \wedge d x_{3}+\operatorname{Im}(\bar{x} K) \operatorname{Im}(\bar{x} K) \star d x_{4} \wedge d x_{4}\right) \\
= & -A \wedge \star \phi .
\end{aligned}
$$

Therefore, we obtain $A \wedge \star \phi+\phi \wedge \star A=0$.

Proposition 2.13. For $(A(x), \phi(x))$ defined as in (4), for a Hodge star operator correspond to a rotational invariant metric $h(t) d x \otimes d \bar{x}$, we have $d_{A} \star \phi=0$.

Proof. By definition,

$$
d_{A} \star \phi=d \star \phi+A \wedge \star \phi+\star \phi \wedge A .
$$

First, we compute $d \star \phi=0$.

Take $\star_{E}$ to be the Hodge star operator correspond to the Euclidean metric in $\mathbb{R}^{4}$, then $\star d x_{i}=h(t)^{2} \star_{E} d x_{i}$.

By (4), we have

$$
\begin{aligned}
\phi & =g \operatorname{Im}(\bar{x} d x) \\
& =g\left(\operatorname{Im}(\bar{x}) d x_{1}+\operatorname{Im}(\bar{x} I) d x_{2}+\operatorname{Im}(\bar{x} J) d x_{3}+\operatorname{Im}(\bar{x} K) d x_{4}\right) .
\end{aligned}
$$

Therefore, we calculate

$$
\begin{aligned}
& d \star_{\phi} \\
= & d\left(g h^{2}\right)\left(\left(\operatorname{Im}(\bar{x}) \star_{E} d x_{1}+\operatorname{Im}(\bar{x} I) \star_{E} d x_{2}+\operatorname{Im}(\bar{x} J) \star_{E} d x_{3}+\operatorname{Im}(\bar{x} K) \star_{E} d x_{4}\right)\right) \\
& +g d\left(\operatorname{Im}(\bar{x}) \star_{E} d x_{1}+\operatorname{Im}(\bar{x} I) \star_{E} d x_{2}+\operatorname{Im}(\bar{x} J) \star_{E} d x_{3}+\operatorname{Im}(\bar{x} K) \star_{E} d x_{4}\right) \\
= & \frac{\partial\left(g h^{2}\right)}{\partial x_{1}} \operatorname{Im}(\bar{x}) d x_{1} \wedge \star_{E} d x_{1}+\frac{\partial\left(g h^{2}\right)}{\partial x_{2}} \operatorname{Im}(\bar{x} I) d x_{2} \wedge \star_{E} d x_{2} \\
& +\frac{\partial\left(g h^{2}\right)}{\partial x_{3}} \operatorname{Im}(\bar{x} J) d x_{3} \wedge \star_{E} d x_{3}+\frac{\partial\left(g h^{2}\right)}{\partial x_{4}} \operatorname{Im}(\bar{x} K) d x_{4} \wedge \star_{E} d x_{4}
\end{aligned}
$$

(by Lemma 2.11)

$=2\left(g h^{2}\right)^{\prime}\left(x_{1} \operatorname{Im}(\bar{x})+x_{2} \operatorname{Im}(\bar{x} I)+x_{3} \operatorname{Im}(\bar{x} J)+x_{4} \operatorname{Im}(\bar{x} K)\right) d x_{1} \wedge d x_{2} \wedge d x_{3} \wedge d x_{4}$

(by Lemma 2.10)

$=0$. 
Combining this with Lemma 2.12, we finish the proof.

\subsection{ODEs from the Kapustin-Witten equations}

Recall that the topological twisted equations (1) are equivalent to the following:

$$
\begin{aligned}
F_{A}^{+}-(\phi \wedge \phi)^{+} & =-\lambda\left(d_{A} \phi\right)^{+} \\
F_{A}^{-}-(\phi \wedge \phi)^{-} & =\lambda^{-1}\left(d_{A} \phi\right)^{-} \\
d_{A}^{\star} \phi & =0 .
\end{aligned}
$$

By Proposition 2.13, we know that $d_{A}^{\star} \phi=0$ is always satisfied under our assumption (4).

Combining Lemma 2.7 and 2.9, we obtain the following ODEs:

$$
\left\{\begin{array}{l}
f^{\prime}+\lambda g^{\prime}+f^{2}-g^{2}+2 \lambda f g=0 \\
t f^{\prime}-t \lambda^{-1} g^{\prime}+2 f-2 \lambda^{-1} g+g^{2} t-f^{2} t+2 t f g \lambda^{-1}=0 .
\end{array}\right.
$$

To summarize the previous computation, we have the following theorem:

Theorem 2.14. Given a solution $(f(t), g(t))$ to the ODEs (6), taking $A(x)=\operatorname{Im}(f(x) \bar{x} d x)$ and $\phi(x)=\operatorname{Im}(g(x) \bar{x} d x)$ gives a solution to the topological twisted equations (1).

By some linear transformations, we obtain the following ODEs:

$$
\left\{\begin{array}{l}
\left(\lambda+\lambda^{-1}\right) t f^{\prime}+2 \lambda f-\left(\lambda-\lambda^{-1}\right)\left(t f^{2}-t g^{2}\right)-2 g+4 f g t=0 \\
\left(\lambda+\lambda^{-1}\right) t g^{\prime}+2 \lambda^{-1} g-2 f+\left(\lambda-\lambda^{-1}\right) 2 f g t+2 t\left(f^{2}-g^{2}\right)=0
\end{array}\right.
$$

Taking $\lambda=-1$, we obtain

$$
\left\{\begin{array}{l}
t f^{\prime}+f+g-2 f g t=0 \\
t g^{\prime}+g+f-t\left(f^{2}-g^{2}\right)=0
\end{array}\right.
$$

We call the equations (8) the Kapustin-Witten ODEs.

Remark. The equations (7) are degenerate at $t=0$, which means that we may not have the uniqueness theorem for a given initial value. Given a solution $(f(t), g(t))$ to (7), if we assume $(f(t), g(t))$ is continous near $t=0$ and $\lim _{t \rightarrow 0}\left(t f^{\prime}(t), t g^{\prime}(t)\right)=(0,0)$, we can take $t \rightarrow 0$ in both sides of the equations (7) and we obtain that $\lambda f(0)=g(0)$. 


\section{Explicit solutions for ODEs}

In this section, we will discuss some properties of the equations (7) and explicitly solve the equations (7) for some special cases.

\subsection{Change of variables}

We are going to simplify the equations (7) by a change of variables.

Take $\tilde{f}(t):=t f(t), \tilde{g}(t):=t g(t)$, then the equations (7) become

$$
\left\{\begin{array}{l}
\frac{\lambda+\lambda^{-1}}{2} t \tilde{f}^{\prime}=\frac{\lambda-\lambda^{-1}}{2}\left(\tilde{f}^{2}-\tilde{g}^{2}-\tilde{f}\right)+\tilde{g}-2 \tilde{f} \tilde{g} \\
\frac{\lambda+\lambda^{-1}}{2} t \tilde{g}^{\prime}=\frac{\lambda-\lambda^{-1}}{2}(\tilde{g}-2 \tilde{f} \tilde{g})+\left(\tilde{f}-\tilde{f}^{2}+\tilde{g}^{2}\right) .
\end{array}\right.
$$

Remark. It is easy to see that if $(f, g)$ is a solution for some parameter $\lambda_{0}$, then $(f,-g)$ is a solution for $-\lambda_{0}$. This is compatible with changing the orientation of the manifold in the topological twisted equation (1).

Taking $u(t):=\tilde{f}(t)-\frac{1}{2}, v(t):=\tilde{g}(t)$, we obtain

$$
\left\{\begin{array}{l}
\frac{\lambda+\lambda^{-1}}{2} t u^{\prime}=\frac{\lambda-\lambda^{-1}}{2}\left(u^{2}-v^{2}-\frac{1}{4}\right)-2 u v \\
\frac{\lambda+\lambda^{-1}}{2} t v^{\prime}=-\frac{\lambda-\lambda^{-1}}{2} 2 u v-\left(u^{2}-v^{2}-\frac{1}{4}\right) .
\end{array}\right.
$$

In order to obtain an autonomous ODE systems, we take $\tilde{u}(s):=u\left(e^{s}\right)$ and $\tilde{v}(s):=v\left(e^{s}\right)$. We obtain

$$
\left\{\begin{array}{l}
\frac{\lambda+\lambda^{-1}}{2} \tilde{u}^{\prime}=\frac{\lambda-\lambda^{-1}}{2}\left(\tilde{u}^{2}-\tilde{v}^{2}-\frac{1}{4}\right)-2 \tilde{u} \tilde{v} \\
\frac{\lambda+\lambda^{-1}}{2} \tilde{v}^{\prime}=-\frac{\lambda-\lambda^{-1}}{2} 2 \tilde{u} \tilde{v}-\left(\tilde{u}^{2}-\tilde{v}^{2}-\frac{1}{4}\right) .
\end{array}\right.
$$

Here $\tilde{u}^{\prime}:=\frac{d \tilde{u}(s)}{d s}$ and $\tilde{v}^{\prime}:=\frac{d \tilde{v}(s)}{d s}$.

\subsection{Some basic properties}

Even though the equations (11) are non-linear, we can find a first integral which can simplify the equations in some special cases. 
Proposition 3.1. Given $(\tilde{u}, \tilde{v})$ a solution to the equations 11 , $I(\tilde{u}, \tilde{v})=$ $\frac{1}{3} \tilde{u}^{3}-\tilde{u} \tilde{v}^{2}-\frac{1}{4} \tilde{u}-\frac{\lambda-\lambda^{-1}}{2}\left(\tilde{v}^{3}-\tilde{u}^{2} \tilde{v}+\frac{1}{4} \tilde{v}\right)$ is a constant.

Proof. We calculate that

$$
\begin{aligned}
\left(\frac{1}{3} \tilde{u}^{3}-\tilde{u} \tilde{v}^{2}-\frac{1}{4} \tilde{u}\right)^{\prime} & =\tilde{u}^{2} \tilde{u}^{\prime}-\tilde{u}^{\prime} \tilde{v}^{2}-2 \tilde{u} \tilde{v} \tilde{v}^{\prime}-\frac{1}{4} \tilde{u}^{\prime} \\
& =\tilde{u}^{\prime}\left(\tilde{u}^{2}-\tilde{v}^{2}-\frac{1}{4}\right)-2 \tilde{u} \tilde{v} \tilde{v}^{\prime} \\
& =\frac{\lambda-\lambda^{-1}}{\lambda+\lambda^{-1}}\left(\left(\tilde{u}^{2}-\tilde{v}^{2}-\frac{1}{4}\right)^{2}+(2 \tilde{u} \tilde{v})^{2}\right)
\end{aligned}
$$

We calculate that

$$
\begin{aligned}
\left(\tilde{v}^{3}-\tilde{u}^{2} \tilde{v}+\frac{1}{4} \tilde{v}\right)^{\prime} & =\tilde{v}^{2} \tilde{v}^{\prime}-\tilde{v}^{\prime} \tilde{u}^{2}-2 \tilde{v} \tilde{u} \tilde{u}^{\prime}+\frac{1}{4} \tilde{v}^{\prime} \\
& =-\left(\tilde{u}^{2}-\tilde{v}^{2}-\frac{1}{4}\right) \tilde{v}^{\prime}-\tilde{u}^{\prime}(2 \tilde{u} \tilde{v}) \\
& =\frac{2}{\lambda+\lambda^{-1}}\left(\left(\tilde{u}^{2}-\tilde{v}^{2}-\frac{1}{4}\right)^{2}+(2 \tilde{u} \tilde{v})^{2}\right)
\end{aligned}
$$

The proposition follows immediately.

Since we would like our solution to exist near $t=0$, recalling that $\tilde{u}(s)=$ $e^{s} f\left(e^{s}\right)-\frac{1}{2}, \tilde{v}(s)=e^{s} g\left(e^{s}\right)$, we obtain the following restrictions:

$$
\lim _{s \rightarrow-\infty} \tilde{u}(s)=-\frac{1}{2} \text { and } \lim _{s \rightarrow-\infty} \tilde{v}(s)=0 .
$$

Therefore, combining this with Proposition 3.1, we have the following identity:

$$
\frac{1}{3} \tilde{u}^{3}-\tilde{u} \tilde{v}^{2}-\frac{1}{4} \tilde{u}-\frac{\lambda-\lambda^{-1}}{2}\left(\tilde{v}^{3}-\tilde{u}^{2} \tilde{v}+\frac{1}{4} \tilde{v}\right)=\frac{1}{12}
$$

By Proposition 3.1, we can prove the following:

Proposition 3.2. For $\lambda \neq \pm 1$, if $f(t)$ does not blow-up in finite time, then $g(t)$ will not blow-up in finite time. 
Proof. By the identity (13), we have

$$
\frac{1}{3} \tilde{u}^{3}-\frac{1}{4} \tilde{u}=\frac{\lambda-\lambda^{-1}}{2}\left(\tilde{v}^{3}-\tilde{u}^{2} \tilde{v}+\frac{1}{4} \tilde{v}\right)+\tilde{u} \tilde{v}^{2}+\frac{1}{12} .
$$

If $f(t)$ does not blow-up in finite time, $\tilde{u}$ will also not blow-up in finite time. If $\tilde{v}$ blows-up in finite time then the right hand side of the identity will be unbounded but the left hand side will be bounded, which gives a contradiction.

Even though the topological twisted equations (1) are not conformally invariant, we still have that it is invariant under rescaling by a constant, which leads to the following proposition:

Proposition 3.3. If $\left(f_{0}(t), g_{0}(t)\right)$ is a solution of the equations (7), then for any constant $C,\left(C f_{0}(C t), C g_{0}(C t)\right)$ are solutions to the equations (7).

Proof. By a direct computation.

\section{3. t'Hooft solution when $\lambda=0$}

In this subsection, we will prove that we can obtain the t'Hooft solution of Yang-Mills equation from the equations (7). By taking $\lambda=0$, (1) becomes

$$
\begin{aligned}
\left(F_{A}-\phi \wedge \phi\right)^{+} & =0 \\
\left(d_{A} \phi\right)^{-} & =0 \\
d_{A}^{*} \phi & =0 .
\end{aligned}
$$

If $\phi=0$, then we are just considering the anti-self dual equation

$$
F_{A}^{+}=0 .
$$

By taking $\lambda=0$, (7) becomes

$$
\left\{\begin{array}{l}
f^{\prime}+f^{2}-g^{2}=0 \\
g^{\prime} t+2 g-2 t f g=0 .
\end{array}\right.
$$

By Theorem 2.12, we know that every solution to the equations (15) will give a solution for the $S L(2 ; \mathbb{C})$ anti-self-dual equation. 
If $g=0$, the equations (15) have a solution $(f(t), g(t))=\left(\frac{1}{1+t}, 0\right)$. The corresponding gauge fields are $\left(A(x)=\operatorname{Im}\left(\frac{1}{1+|x|^{2}} \bar{x} d x\right), \phi(x)=0\right)$, which recovers the t'Hooft solution for anti-self-dual equation in [3].

Remark. We can also find a solution using the first integral $I(\tilde{u}, \tilde{v})=$ $\tilde{v} \tilde{u}^{2}-\frac{1}{3} \tilde{v}^{3}-\frac{1}{4} \tilde{v}$. After some computation, we obtain the solution $(f(t), g(t))$ $=\left(\frac{t}{t^{2}-1}, \frac{\sqrt{3}}{t^{2}-1}\right)$ to 15 .

\subsection{Explicit solutions to the Kapustin-Witten ODEs}

Taking $\lambda=-1$, the equations (7) become

$$
\left\{\begin{array}{l}
t f^{\prime}+f+g-2 f g t=0 \\
t g^{\prime}+f+g-t\left(f^{2}-g^{2}\right)=0
\end{array}\right.
$$

We can find a solution

$$
\left\{\begin{array}{l}
f(t)=\frac{1}{2 t} \\
g(t)=\frac{\tan \left(-\frac{1}{2} \ln (t)+C\right)}{2 t}
\end{array}\right.
$$

However, the solution will have so many poles that 0 will be an accumulation point of singularities, which is not what we want. We hope to find a solution which is well-defined near 0 .

From the equations (10), we obtain the ODEs corresponding to the Kapustin-Witten equations:

$$
\left\{\begin{array}{l}
t u^{\prime}=2 u v \\
t v^{\prime}=u^{2}-v^{2}-\frac{1}{4}
\end{array}\right.
$$

Recalling that $u(t)=t f(t)-\frac{1}{2}$ and $v(t)=t g(t)$, we hope to obtain a solution well-defined near $t=0$. Therefore, we hope to solve (18) for the initial value $\left(u(0)=-\frac{1}{2}, v(0)=0\right)$. 
By taking $\tilde{u}(s):=u\left(e^{s}\right), \tilde{v}(s):=v\left(e^{s}\right)$, we obtain an autonomous system of ODEs:

$$
\left\{\begin{array}{l}
\tilde{u}^{\prime}=2 \tilde{u} \tilde{v} \\
\tilde{v}^{\prime}=\tilde{u}^{2}-\tilde{v}^{2}-\frac{1}{4} \\
\lim _{s \rightarrow-\infty} \tilde{u}(s)=-\frac{1}{2} \\
\lim _{s \rightarrow-\infty} \tilde{v}(s)=0 .
\end{array}\right.
$$

By Proposition 3.1, we the following identity:

$$
\tilde{v}^{2} \tilde{u}-\frac{1}{3} \tilde{u}^{3}+\frac{1}{4} \tilde{u}=-\frac{1}{12} .
$$

Combining (19) and (20), we obtain

$$
\left\{\begin{array}{l}
12 \tilde{v}^{2} \tilde{u}=(2 \tilde{u}+1)^{2}(\tilde{u}-1) \\
\tilde{u}^{\prime}=2 \tilde{u} \tilde{v} \\
\lim _{s \rightarrow-\infty} \tilde{u}(s)=-\frac{1}{2} \\
\lim _{s \rightarrow-\infty} \tilde{v}(s)=0 .
\end{array}\right.
$$

Assuming $\tilde{u}<0$, take $W(s):=-\tilde{u}(s)$, so we are trying to solve the following ODEs:

$$
W(s)^{\prime}= \pm \frac{1}{\sqrt{3}} \sqrt{W(s)} \sqrt{W(s)+1}(2 W(s)-1) .
$$

We first solve $W(s)^{\prime}=\frac{1}{\sqrt{3}} \sqrt{W(s)} \sqrt{W(s)+1}(2 W(s)-1)$.

Taking $H(s):=\frac{1+4 W(s)}{2 \sqrt{3} \sqrt{W(s)^{2}+W(s)}}$, we have the following Lemma:

Lemma 3.4. $\frac{1}{1-H^{2}(s)} d H(s)=-d s$

Proof. We calculate that

$$
H^{\prime}(s)=\frac{(2 W(s)-1) W(s)^{\prime}}{4 \sqrt{3}\left(W(s)^{2}+W(s)\right) \sqrt{W(s)^{2}+W(s)}} .
$$

In addition, we calculate that

$$
1-H(s)^{2}=-\frac{(2 W(s)-1)^{2}}{12\left(W(s)^{2}+W(s)\right)} .
$$


Combining this with $W(s)^{\prime}=\frac{1}{\sqrt{3}} \sqrt{W(s)} \sqrt{W(s)+1}(2 W(s)-1)$, the result follows immediately.

By the previous lemma, $\frac{1}{2} \ln \left(\frac{H(s)+1}{H(s)-1}\right)=-s+C$. Therefore, $H(s)=\frac{C e^{-2 s}+1}{C e^{-2 s}-1}$. Combining this with $H(s)=\frac{1+4 W(s)}{2 \sqrt{3} \sqrt{W(s)^{2}+W(s)}}$, we find

$$
W(s)=\frac{2-3 H^{2}+3 H \sqrt{H^{2}-1}}{2\left(3 H^{2}-4\right)} .
$$

Therefore, we have

$$
W(\ln t)=\frac{1}{2} \frac{C^{2} t^{2}-2 C t+1}{C^{2} t^{2}+4 C t+1}
$$

By definition,

$$
f(t)=\frac{\frac{1}{2}-W(\ln t)}{t} .
$$

We calculate that

$$
f(t)=\frac{3 C}{C^{2} t^{2}+4 C t+1}(\text { for any constant C). }
$$

Putting this into the equations 19 and taking $g(t)=\frac{\tilde{v}(\ln (t))}{t}$, we obtain

$$
g(t)=\frac{3 C(C t+1)}{\left(C^{2} t^{2}+4 C t+1\right)(C t-1)} .
$$

For $W(s)^{\prime}=-\frac{1}{\sqrt{3}} \sqrt{W(s)} \sqrt{W(s)+1}(2 W(s)-1)$, we obtain another solution $(f(t), g(t))=\left(\frac{1}{t} \frac{C^{2} t^{2}+C t+1}{C^{2} t^{2}+4 C t+1},-\frac{3 C(C t-1)}{\left(C^{2} t^{2}+4 C t+1\right)(C t+1)}\right)$.

To summarize, by solving the equations (7) with $\lambda=-1$, we obtain the following proposition:

\section{Proposition 3.5.}

$$
\left\{\begin{aligned}
f_{1}(t) & =\frac{3 C}{(C t)^{2}+4(C t)+1} \\
g(t) & =\frac{3 C(C t+1)}{\left((C t)^{2}+4(C t)+1\right)(C t-1)},
\end{aligned}\right.
$$




$$
\left\{\begin{aligned}
f_{2}(t) & =\frac{1}{t} \frac{C^{2} t^{2}+C t+1}{C^{2} t^{2}+4 C t+1} \\
g(t) & =\frac{3 C(C t+1)}{\left((C t)^{2}+4(C t)+1\right)(C t-1)}
\end{aligned}\right.
$$

are two families of solutions to the Kapustin-Witten ODEs (8).

\section{Instanton number zero solutions}

In this section, we will give a complete proof of Theorem 1.1.

\subsection{Computation of instanton numbers}

We will now give a formula to compute the instanton number for the rotationally invariant solutions, which will prove property (2) of Theorem 1.1.

Lemma 4.1. $\bar{x} d x \wedge d \bar{x} x \wedge \bar{x} d x \wedge d \bar{x} x=24 t^{2} d x_{1} \wedge d x_{2} \wedge d x_{3} \wedge d x_{4}, \quad d \bar{x} \wedge$ $d x \wedge d \bar{x} \wedge d x=-24 d x_{1} \wedge d x_{2} \wedge d x_{3} \wedge d x_{4}$.

Proof. By Lemma 2.6, it is just a direct computation.

Combining this with Lemma 2.7, we obtain that

$$
\begin{aligned}
& \left|F_{A}^{-}\right|^{2}=6\left(t f^{\prime}+2 f-t f^{2}\right)^{2} \\
& \left|F_{A}^{+}\right|^{2}=6\left(f^{\prime}+f^{2}\right)^{2} t^{2} .
\end{aligned}
$$

Since we are considering the solutions over the non-compact space $\mathbb{R}^{4}$, the instanton number is defined as:

Definition 4.2. Given that a connection $(A(x), \phi(x))$ is a solution to the Kapustin-Witten equations (2), if the integral $\frac{1}{4 \pi^{2}} \int_{\mathbb{R}^{4}} \operatorname{tr}\left(F_{A} \wedge F_{A}\right)$ exist, we define the instanton number $k$ for $(A(x), \phi(x))$ is

$$
k:=\frac{1}{4 \pi^{2}} \int_{\mathbb{R}^{4}} \operatorname{tr}\left(F_{A} \wedge F_{A}\right) \in \mathbb{R} .
$$

For a rotationally invariant solution as in (4), we have a simple formula to compute the instanton number. 
Proposition 4.3. For a globally defined $C^{1}$ connection $A(x)=\operatorname{Im}(f(t) \bar{x} d x)$ over $\mathbb{R}^{4}$, by taking $\tilde{f}(t):=t f(t)$, the instanton number $k$ satisfies:

$$
k=6 \int_{0}^{+\infty} \tilde{f}(\tilde{f}-1) \tilde{f}^{\prime} d t=\left.\left(2 \tilde{f}^{3}-3 \tilde{f}^{2}\right)\right|_{0} ^{+\infty} .
$$

Proof. We calculate that

$$
\begin{aligned}
k= & \frac{1}{4 \pi^{2}} \int_{\mathbb{R}^{4}} \operatorname{tr}\left(F_{A} \wedge F_{A}\right) \\
= & \frac{1}{4 \pi^{2}} \int_{\mathbb{R}^{4}}\left(\left|F_{A}^{+}\right|^{2}-\left|F_{A}^{-}\right|^{2}\right) d x_{1} \wedge d x_{2} \wedge d x_{3} \wedge d x_{4} \\
= & \frac{1}{4 \pi^{2}} \int_{\mathbb{R}^{4}}\left(6\left(f^{\prime}+f^{2}\right)^{2} t^{2}-6\left(t f^{\prime}+2 f-t f^{2}\right)^{2}\right) d x_{1} \wedge d x_{2} \wedge d x_{3} \wedge d x_{4} \\
= & \frac{1}{4 \pi^{2}} \int_{\mathbb{R}^{4}} 24\left(t f^{2}-f\right)\left(t f^{\prime}+f\right) d x_{1} \wedge d x_{2} \wedge d x_{3} \wedge d x_{4} \\
= & \frac{1}{4 \pi^{2}} \int_{\mathbb{R}^{4}} 24\left(\frac{1}{t} \tilde{f}(\tilde{f}-1) \tilde{f}^{\prime}\right) d x_{1} \wedge d x_{2} \wedge d x_{3} \wedge d x_{4} \\
= & \frac{1}{4 \pi^{2}} 12 \operatorname{Vol}\left(S^{3}\right) \int_{0}^{+\infty}\left(\tilde{f}(\tilde{f}-1) \tilde{f}^{\prime}\right) d t \\
& \left(\operatorname{by} d x_{1} \wedge d x_{2} \wedge d x_{3} \wedge d x_{4}=d \operatorname{Vol}_{S^{3}} \frac{1}{2} t d t\right) \\
= & 6 \int_{0}^{+\infty}\left(\tilde{f}(\tilde{f}-1) \tilde{f}^{\prime}\right) d t \quad\left(\text { since } \operatorname{Vol}\left(S^{3}\right)=2 \pi^{2}\right) \\
= & \left.\left(2 \tilde{f}^{3}-3 \tilde{f}^{2}\right)\right|_{0} ^{+\infty} .
\end{aligned}
$$

Remark. The previous formula for the instanton number only works for connections with the specific type $A(x)=\operatorname{Im}(f(t) \bar{x} d x)$.

For $A(x)=\operatorname{Im}(f(t) x d \bar{x})$, a conjugate form of (4), we have the following corollary:

Corollary 4.4. For a globally defined $C^{1}$ connection $A=\operatorname{Im}(f(t) \bar{x} d x)$ over $\mathbb{R}^{4}$, by taking $\tilde{f}(t):=t f(t)$, the instanton number $k$ satisfies:

$$
k=-6 \int_{0}^{+\infty} \tilde{f}(\tilde{f}-1) \tilde{f}^{\prime} d t=\left.\left(3 \tilde{f}^{2}-2 \tilde{f}^{3}\right)\right|_{0} ^{+\infty}
$$


Proof. We can calculate in a similar way and obtain that

$$
\begin{aligned}
& \left|F_{A}^{+}\right|^{2}=6\left(t f^{\prime}+2 f-t f^{2}\right)^{2} \\
& \left|F_{A}^{-}\right|^{2}=6\left(f^{\prime}+f^{2}\right)^{2} t^{2} .
\end{aligned}
$$

By the same computation as in Proposition 4.3, we obtain the result.

Corollary 4.5. The solution $\left(f_{1}(t), g(t)\right)=\left(\frac{3}{t^{2}+4 t+1}, \frac{3(t+1)}{\left(t^{2}+4 t+1\right)(t-1)}\right)$ to the Kapustin-Witten ODEs (8) has instanton number zero.

The solution $\left(f_{2}(t), g(t)\right)=\left(\frac{1}{t} \frac{t}{2}+t+1^{t^{2}+4 t+1}, \frac{3(t+1)}{\left(t^{2}+4 t+1\right)(t-1)}\right)$ to the KapustinWitten ODEs (8) has instanton number zero.

Proof. Defining $\tilde{f}_{1}(t):=t f_{1}(t)$, then $\tilde{f}_{1}(0)=\tilde{f}_{1}(+\infty)=0$. Therefore, by Proposition 4.3. we know the instanton number of $A(x)=\operatorname{Im}\left(f_{1}(t) \bar{x} d x\right)$ is 0 .

Similarly, for $\tilde{f}_{2}(t):=t f_{2}(t)$, we have $\tilde{f}_{2}(0)=\tilde{f}_{2}(+\infty)=1$. Therefore, by Proposition 4.3, we know the instanton number of $A(x)=\operatorname{Im}\left(f_{2}(t) \bar{x} d x\right)$ is 0 .

Proposition 4.6. Given that $(\tilde{f}(t), \tilde{g}(t))$ is a solution to the equations (9), if

(1) $\lim _{t \rightarrow 0} \tilde{f}(t)$ and $\lim _{t \rightarrow 0} \tilde{g}(t)$ exist.

(2) $\lim _{t \rightarrow+\infty} \tilde{f}(t)$ and $\lim _{t \rightarrow+\infty} \tilde{g}(t)$ exist.

Then $A(x)=\operatorname{Im}\left(\frac{\tilde{f}(t)}{t} \bar{x} d x\right)$ is a connection with instanton number 0,1 or -1 .

Proof. After a change of variable and translation, the ODEs (9) turn into (11). Equation (11) is an autonomous system, therefore the limit point must be a equilibrium point of (11).

There are two equilibrium points, $(\tilde{u}, \tilde{v}) \in\left\{\left(\frac{1}{2}, 0\right),\left(-\frac{1}{2}, 0\right)\right\}$ or equivalently $(\tilde{f}, \tilde{g}) \in\{(0,0),(1,0)\}$. Therefore, $(\tilde{f}(0), \tilde{g}(0)) \in\{(0,0),(1,0)\}$ and $(\tilde{f}(+\infty), \tilde{g}(+\infty)) \in\{(0,0),(1,0)\}$. By Proposition 4.3 , we know that $A(x)=$ $\operatorname{Im}\left(\frac{\tilde{f}(t)}{t} \bar{x} d x\right)$ can only have instanton number 0,1 or -1 .

\subsection{Bubbling for instanton number 0 singular solutions to the Kapustin-Witten equations}

In this subsection, we will prove property (3) of Theorem 1.1: the existence of some bubbling phenomenon for singular solutions. 
By previous computation, we know that:

$$
\begin{aligned}
& \left|F_{A}^{-}\right|^{2}=6\left(t f^{\prime}+2 f-t f^{2}\right)^{2} \\
& \left|F_{A}^{+}\right|^{2}=6\left(f^{\prime}+f^{2}\right)^{2} t^{2}
\end{aligned}
$$

Consider the solution

$$
\left\{\begin{array}{l}
f(t)=\frac{3}{t^{2}+4 t+1} \\
g(t)=\frac{3(t+1)}{\left(t^{2}+4 t+1\right)(t-1)} .
\end{array}\right.
$$

Combining (26) and (27), we obtain that

$$
\begin{aligned}
\left|F_{A}\right|^{2} & =\left|F_{A}^{+}\right|^{2}+\left|F_{A}^{-}\right|^{2} \\
& =\left(6\left(t f^{\prime}+2 f-t f^{2}\right)^{2}+6\left(f^{\prime}+f^{2}\right)^{2} t^{2}\right) \\
& =\frac{108\left(2 t^{4}+2 t^{3}+t^{2}+2 t+2\right)}{\left(t^{2}+4 t+1\right)^{4}} .
\end{aligned}
$$

As the curvature norm $\left|F_{A}\right|$ plays an important roles in the Uhlenbeck compactness theorem [4] [11, we also hopes to understand it in the KapustinWitten equations.

The graph of $\left|F_{A}\right|(t)$ is depicted in Figure 1:

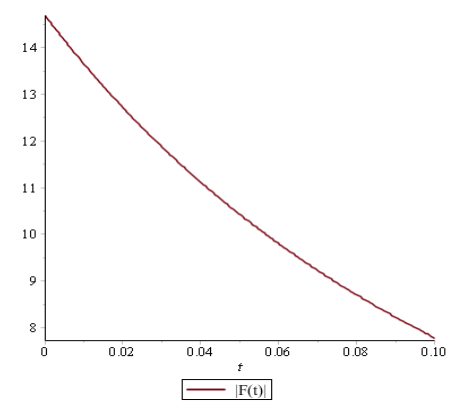

Figure 1: Norm of $\left|F_{A}\right|$ as a Function of Radius $t$.

Proposition 4.7. $\left|F_{A}\right|(t)$ is decreasing and $\left|F_{A}\right|(0)$ is its maximum. 
Proof. A direct computation shows that

$$
\frac{d}{d t}\left|F_{A}\right|^{2}(t)=-\frac{216\left(4 t^{5}+5 t^{4}+3 t^{3}+8 t^{2}+19 t+15\right)}{\left(t^{2}+4 t+1\right)^{5}},
$$

so $\left|F_{A}\right|^{\prime}<0$ for all $t \geq 0$. Therefore, $\left|F_{A}\right|$ is decreasing and $\left|F_{A}\right|(0)$ is its maximum.

By Proposition 3.5, for any constant C, we have the solutions

$$
\left\{\begin{array}{l}
f^{C}(t)=\frac{3 C}{C^{2} t^{2}+4 C t+1} \\
g^{C}(t)=\frac{3 C(C t+1)}{\left(C^{2} t^{2}+4 C t+1\right)(C t-1)}
\end{array}\right.
$$

to the Kapustin-Witten ODEs.

We define $\left|F_{A}^{C}\right|(t)$ as the curvature norm for $f^{C}(t)$, then we have the following proposition:

Proposition 4.8. (1) $\left|F_{A}^{C}\right|(t)=C\left|F_{A}\right|(C t)$.

(2) $\int_{\mathbb{R}^{4}}\left|F_{A}^{C}\right|^{2}(t) d \mathrm{Vol}=\int_{\mathbb{R}^{4}}\left|F_{A}\right|^{2}(t) d \mathrm{Vol}$.

Proof. For (1), this is an immediately computation. For (2), by the definition of $t=|x|^{2}$, we get the result immediately.

Proposition 4.9. Let $D$ to be a real number defines as follows: $D:=$ $\int_{\mathbb{R}^{4}}\left|F_{A}^{C}\right|^{2}(t) d \mathrm{Vol}$. Then we have $\lim _{C \rightarrow+\infty} \frac{1}{D}\left|F_{A}^{C}\right|(t)=\delta_{0}$, where $\delta_{0}$ is the Dirac measure at 0 .

Proof. By Proposition 4.8, we know that

$$
\frac{1}{D}\left|F_{A}^{C}\right|(t)=\frac{C}{D}\left|F_{A}\right|(C t) \quad \text { and } \quad \int_{\mathbb{R}^{4}} \frac{1}{D}\left|F_{A}^{C}\right|^{2}(t) d \mathrm{Vol}=1 .
$$

Therefore, the function $\frac{C}{D}\left|F_{A}\right|(C t)$ is a rescale of $\left|F_{A}\right|(t)$ and has integral 1. By classical approximations to the identity results [8], we obtain $\lim _{C \rightarrow+\infty} \frac{1}{D}\left|F_{A}^{C}\right|(t)=\delta_{0}$.

\subsection{Non-removability of singularities for $\phi$ by $\mathrm{SU}(2)$ gauge transformations}

In this subsection, we will prove property (4) of Theorem 1.1. It is suffice to consider the $C=1$ case. 
By Lemma 2.9, we obtain that

$$
\begin{aligned}
\left(d_{A} \phi\right)^{+} & =-\frac{1}{2}\left(g^{\prime}+2 f g\right) \bar{x} d x \wedge d \bar{x} x \\
& =\frac{3\left(t^{3}+3 t+2\right)}{\left(t^{2}+4 t+1\right)(t-1)\left(t^{3}+3 t^{2}-3 t+1\right)} \bar{x} d x \wedge d \bar{x} x, \\
\left(d_{A} \phi\right)^{-} & =\left(\frac{1}{2} g^{\prime} t+g-f g t\right) d \bar{x} \wedge d x \\
& =-3 \frac{2 t^{3}+3 t^{2}+1}{\left(t^{2}+4 t+1\right)(t-1)\left(t^{3}+3 t^{2}-3 t+1\right)} d \bar{x} \wedge d x .
\end{aligned}
$$

We calculate that

$$
\left|d_{A} \phi\right|^{2}=\frac{432\left(2 t^{8}+6 t^{7}+5 t^{6}+2 t^{5}+6 t^{4}+2 t^{3}+5 t^{2}+6 t+2\right)}{\left(t^{2}+4 t+1\right)^{2}(t-1)^{2}\left(t^{3}+3 t^{2}-3 t+1\right)^{2}} .
$$

Therefore, we know $\left\|d_{A} \phi\right\|_{L^{2}}$ is unbounded near $t=1$.

Since $\left\|d_{A} \phi\right\|_{L^{2}}$ is invariant under the $S U(2)$ gauge action, we know that the singularities of $\phi$ can not be removed by $S U(2)$ gauge transformations.

\section{Non-zero instanton number solutions}

\subsection{Instanton number \pm 1 solutions}

In this subsection, we are going to give a proof of Theorem 1.2.

First, we are going to give a construction of an instanton number 1 solution.

By Proposition 4.3, we know that the instanton number is determined by the limit behavior of our connection $A(x)=\operatorname{Im}(f(t) \bar{x} d x)$. In order to construct an instanton number \pm 1 solution, we only need to construct a solution with different equilibrium points at $t=0$ and $t=+\infty$.

By Proposition 3.5, taking $C=1$, we have the following solutions:

$$
\left\{\begin{array}{l}
f_{1}(t)=\frac{3}{t^{2}+4 t+1} \\
g_{1}(t)=\frac{3(t+1)}{\left(t^{2}+4 t+1\right)(t-1)},
\end{array}\right.
$$


(30)

$$
\left\{\begin{array}{l}
f_{2}(t)=\frac{1}{t} \frac{t^{2}+t+1}{t^{2}+4 t+1} \\
g_{2}(t)=\frac{3(t+1)}{\left(t^{2}+4 t+1\right)(t-1)}
\end{array}\right.
$$

As $g_{1}(t)=g_{2}(t)$, we hope to understand the relationship of $f_{1}(t)$ and $f_{2}(t)$. The graphs of $f_{1}(t)$ and $f_{2}(t)$ are depicted in Figure 2 and obviously, we get $f_{1}(1)=f_{2}(1)$.

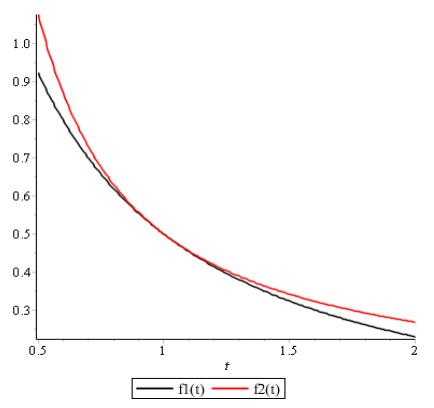

Figure 2: Graph of $f_{1}(t)$ and $f_{2}(t)$.

Now, we hope to glue these two solutions to obtain a new solution.

\section{Proposition 5.1.}

$$
\begin{aligned}
& A(x)=\left\{\begin{array}{l}
\operatorname{Im}\left(\frac{3}{t^{2}+4 t+1} \bar{x} d x\right) \quad(t \leq 1) \\
\operatorname{Im}\left(\frac{1}{t} \frac{t^{2}+t+1}{t^{4}+4 t+1} \bar{x} d x\right)(t \geq 1)
\end{array}\right. \\
& \phi(t)=\operatorname{Im}\left(\frac{3(t+1)}{(t-1)\left(t^{2}+4 t+1\right)} \bar{x} d x\right)
\end{aligned}
$$

is a solution to the Kapustin-Witten Equations (2) which satisfies the following properties:

(1) The solution has instanton number $=1$.

(2) $A(x)$ is $C^{\infty}$ away from $t=1$ and $C^{1}$ at $t=1, \phi(t)$ is singular at $t=1$. 
Proof.

$$
f(t)=\left\{\begin{array}{c}
\frac{3}{t^{2}+4 t+1}(t \leq 1) \\
\frac{1}{t} \frac{t^{2}+t+1}{t^{4}+4 t+1}(t \geq 1)
\end{array}\right.
$$

Taking $\tilde{f}(t)=t f(t)$, by a direct computation, we know that $\tilde{f}(0)=0$, $\tilde{f}(+\infty)=1$. By Proposition 4.3 , we know that the instanton number is equal to 1 .

Defining $u(t):=\tilde{f}(t)-\frac{1}{2}$, then

$$
u(t)=\left\{\begin{array}{r}
\frac{1}{2} \frac{t^{2}-2 t+1}{t^{2}+4 t+1}(t \leq 1) \\
-\frac{1}{2} \frac{t^{2}-2 t+1}{t^{2}+4 t+1}(t \geq 1) .
\end{array}\right.
$$

By a direct computation, we know that $u(t)$ is a $C^{1}$ function. Therefore, $A(x)$ is also a $C^{1}$ connection.

Remark. By Corollary 4.4, we know

$$
\begin{gathered}
A(x)=\left\{\begin{array}{l}
\operatorname{Im}\left(\frac{3}{t^{2}+4 t+1} x d \bar{x}\right)(t \leq 1) \\
\operatorname{Im}\left(\frac{1}{t} \frac{t^{2}+t+1}{t^{2}+4 t+1} x d \bar{x}\right)(t \geq 1)
\end{array}\right. \\
\phi(x)=\operatorname{Im}\left(\frac{3(t+1)}{(t-1)\left(t^{2}+4 t+1\right)} x d \bar{x}\right)
\end{gathered}
$$

is a instanton number -1 solution to the Kapustin-Witten equations.

\subsection{Linear combination of solutions}

In this subsection, we aim to generalize the ADHM construction from [1] to obtain higher instanton number solutions to the Kapustin-Witten equations. However, there exists an essential problem to generalizing the instanton number computation method from the anti-self-dual equation case [1]. We conjecture that we will obtain some higher instanton number solutions from this construction. 
In view of Corollary 4.4, without loss of generality, we can focus on instanton number $k \geq 0$.

Now, let $\lambda_{1}, \ldots, \lambda_{k}$ be $k$ real numbers and $b_{1}, \ldots, b_{k}$ be $k$ numbers in $\mathbb{H}$. Take $U:=\left(\lambda_{1}\left(x-b_{1}\right), \ldots, \lambda_{k}\left(x-b_{k}\right)\right)^{T}$ and $U^{\star}$ be the conjugate transpose of $U$. Take $e_{0}=1, e_{1}=I, e_{2}=J, e_{3}=K$, then for any quaternion $b_{i}$, we can write $b_{i}=b_{i j} e_{j}$.

Now we are going to compute an identity which is parallel to $k=0$ case.

Lemma 5.2. For any $g(t) \in C^{1}, d \star \operatorname{Im}\left(g\left(|U|^{2}\right) U^{\star} d U\right)=0$

Proof. We calculate that

$$
\begin{aligned}
& d \operatorname{Im}\left(g\left(|U|^{2}\right) \star U^{\star} d U\right) \\
= & \sum_{i=1}^{k} d g\left(|U|^{2}\right) \operatorname{Im}\left(\lambda_{i}^{2}\left(\bar{x}-\bar{b}_{i}\right) \wedge \star d\left(x-b_{i}\right)\right)(\text { by Lemma 2.11) } \\
= & \sum_{i=1}^{k} \sum_{j=1}^{4} d g\left(|U|^{2}\right) \lambda_{i}^{2} \operatorname{Im}\left(\left(\bar{x}-\bar{b}_{i}\right) e_{j}\right) \wedge \star d\left(x_{j}-b_{i j}\right) \\
= & \sum_{i=1}^{k} \lambda_{i}^{2} \sum_{j=1}^{4} \frac{\partial g}{\partial x_{j}} \operatorname{Im}\left(\left(\bar{x}-\bar{b}_{i}\right) e_{j}\right) d x_{j} \wedge \star d x_{j} \\
= & \sum_{i=1}^{k} \lambda_{i}^{2} g^{\prime} \sum_{j=1}^{4} \frac{\partial|U|^{2}}{\partial x_{j}} \operatorname{Im}\left(\left(\bar{x}-\bar{b}_{i}\right) e_{j}\right) d x_{j} \wedge \star d x_{j} \\
= & \sum_{i=1}^{k} \lambda_{i}^{2} g^{\prime} \sum_{l=1}^{k} \lambda_{l}^{2}\left(x_{j}-b_{l j}\right) \sum_{j=1}^{4} \operatorname{Im}\left(\left(\bar{x}-\bar{b}_{i}\right) e_{j}\right) d x_{j} \wedge \star d x_{j} \\
= & 2 g^{\prime} d \operatorname{Vol} \sum_{i=1}^{k} \sum_{l=1}^{k} \sum_{j=1}^{4} \lambda_{i}^{2} \lambda_{l}^{2}\left(x_{j}-b_{l j}\right) \operatorname{Im}\left(\left(\bar{x}-\bar{b}_{i}\right) e_{j}\right) \\
= & g^{\prime} d \operatorname{Vol} \sum_{i=1}^{k} \sum_{l=1}^{k} \sum_{j=1}^{4} \lambda_{i}^{2} \lambda_{l}^{2}\left(\sum_{j=1}^{4}\left(x_{j}-b_{l j}\right) \operatorname{Im}\left(\left(\bar{x}-\bar{b}_{i}\right) e_{j}\right)\right. \\
& \left.+\sum_{j=1}^{4}\left(x_{j}-b_{i j}\right) \operatorname{Im}\left(\left(\bar{x}-\bar{b}_{l}\right) e_{j}\right)\right) .
\end{aligned}
$$

Therefore, in order to show $d \star \operatorname{Im}\left(g\left(|U|^{2}\right) U^{\star} d U\right)=0$, we only need to show

$$
\sum_{j=1}^{4}\left(x_{j}-b_{l j}\right) \operatorname{Im}\left(\left(\bar{x}-\bar{b}_{i}\right) e_{j}\right)+\sum_{j=1}^{4}\left(x_{j}-b_{i j}\right) \operatorname{Im}\left(\left(\bar{x}-\bar{b}_{l}\right) e_{j}\right)=0 .
$$


By translation, without loss of generality, we can assume $b_{i}=0$. Then we calculate that

$$
\begin{aligned}
& \sum_{j=1}^{4}\left(x_{j}-b_{l j}\right) \operatorname{Im}\left((\bar{x}) e_{j}\right) \\
= & \sum_{j=1}^{4} x_{j} \operatorname{Im}\left((\bar{x}) e_{j}\right)-\sum_{j=1}^{4} b_{l j} \operatorname{Im}\left((\bar{x}) e_{j}\right) \\
= & -\sum_{j=1}^{4} b_{l j} \operatorname{Im}\left((\bar{x}) e_{j}\right) .
\end{aligned}
$$

For the rest, we calculate that

$$
\begin{aligned}
& \sum_{j=1}^{4} x_{j} \operatorname{Im}\left(\left(\bar{x}-\bar{b}_{l}\right) e_{j}\right) \\
= & -\sum_{j=1}^{4} x_{j} \operatorname{Im}\left(\bar{b}_{l} e_{j}\right) \\
= & -\left(x_{1}\left(-b_{l 2} I-b_{l 3} J-b_{l 4} K\right)+x_{2}\left(b_{l 1} I+b_{l 3} K-b_{l 4} J\right)\right. \\
& \left.+x_{3}\left(b_{l 1} J-b_{l 2} K+b_{l 4} I\right)+x_{4}\left(b_{l 1} K+b_{l 2} J-b_{l 3} I\right)\right) \\
= & \sum_{j=1}^{4} b_{l j} \operatorname{Im}\left((\bar{x}) e_{j}\right) .
\end{aligned}
$$

Therefore, we obtain the following identity:

$$
\sum_{j=1}^{4}\left(x_{j}-b_{l j}\right) \operatorname{Im}\left(\left(\bar{x}-\bar{b}_{i}\right) e_{j}\right)+\sum_{j=1}^{4}\left(x_{j}-b_{i j}\right) \operatorname{Im}\left(\left(\bar{x}-\bar{b}_{l}\right) e_{j}\right)=0
$$

Combining all the things above, we obtain the lemma.

Lemma 5.3. For any $f(t), g(t) \in C^{1}$, we have

$$
\operatorname{Im}\left(f\left(|U|^{2}\right) U^{\star} d U\right) \wedge \star \operatorname{Im}\left(g\left(|U|^{2}\right) U^{\star} d U\right)=0
$$

Proof. Since $f\left(|U|^{2}\right), g\left(|U|^{2}\right)$ are real functions, we only need to show that $\operatorname{Im}\left(U^{\star} d U\right) \wedge \star \operatorname{Im}\left(U^{\star} d U\right)=0$. 
We calculate that

$$
\begin{aligned}
& \operatorname{Im}\left(U^{\star} d U\right) \wedge \star \operatorname{Im}\left(U^{\star} d U\right) \\
= & \sum_{i=1}^{k} \sum_{j=1}^{4} \lambda_{i}^{2} \operatorname{Im}\left(\left(\bar{x}-\bar{b}_{i}\right) e_{j}\right) d x_{j} \wedge \sum_{m=1}^{k} \sum_{n=1}^{4} \lambda_{m}^{2} \operatorname{Im}\left(\left(\bar{x}-\bar{b}_{m}\right) e_{n}\right) \star d x_{n} \\
= & \sum_{i=1}^{k} \sum_{m=1}^{k} \sum_{j=1}^{4} \lambda_{i}^{2} \lambda_{m}^{2} \operatorname{Im}\left(\left(\bar{x}-\bar{b}_{i}\right) e_{j}\right) \operatorname{Im}\left(\left(\bar{x}-\bar{b}_{m}\right) e_{j}\right) d x_{j} \wedge \star d x_{j} \\
= & -\star \operatorname{Im}\left(U^{\star} d U\right) \wedge \operatorname{Im}\left(U^{\star} d U\right) .
\end{aligned}
$$

Corollary 5.4. For any $f(t), g(t) \in C^{1}$, if $A(x)=\operatorname{Im}\left(f\left(|U|^{2}\right) U^{\star} d U\right)$ and $\phi(x)=\operatorname{Im}\left(g\left(|U|^{2}\right) U^{\star} d U\right)$, then we have $d_{A} \star \phi=0$.

Proof. We have $d_{A} \star \phi=d \phi+A \wedge \star \phi+\phi \wedge \star A$. This is a direct corollary of Lemma 5.2 and Lemma 5.3 .

Taking $f_{1}(t)=\frac{3}{t^{2}+4 t+1}, f_{2}(t)=\frac{1}{t} \frac{t^{2}+t+1}{t^{2}+4 t+1}, g(t)=\frac{3(t+1)}{(t-1)\left(t^{2}+4 t+1\right)}$, we have the following proposition:

\section{Proposition 5.5.}

$$
A(x)=\left\{\begin{array}{l}
\operatorname{Im}\left(f_{1}\left(|U|^{2}\right) U^{\star} d U\right)=\operatorname{Im}\left(\frac{3}{|U|^{4}+4|U|^{2}+1} U^{\star} d U\right) \quad(|U| \leq 1) \\
\operatorname{Im}\left(f_{2}\left(|U|^{2}\right) U^{\star} d U\right)=\operatorname{Im}\left(\frac{1}{|U|^{2}} \frac{|U|^{4}+|U|^{2}+1}{|U|^{4}+4|U|^{2}+1} U^{\star} d U\right) \quad(|U| \geq 1)
\end{array}\right.
$$

$$
\phi(x)=\operatorname{Im}\left(g\left(|U|^{2}\right) U^{\star} d U\right)=\operatorname{Im}\left(\frac{3\left(|U|^{2}+1\right)}{\left(|U|^{2}-1\right)\left(|U|^{4}+4|U|^{2}+1\right)} U^{\star} d U\right)
$$

are solutions to the Kapustin-Witten equations.

Proof. By Corollary 5.4 the equation $d_{A}^{\star} \phi=0$ is always satisfied. Therefore, we only need to show that the equation $F_{A}-\phi \wedge \phi-\star d_{A} \phi=0$ is satisfied by $(A(x), \phi(x))$ defined above.

For

$$
(A(x), \phi(x))=\left(\operatorname{Im}\left(f\left(|U|^{2}\right) U^{\star} d U\right), \operatorname{Im}\left(g\left(|U|^{2}\right) U^{\star} d U\right)\right),
$$

we observe that all the computations in Section 2 can be finish similarly. To be more precise, replacing every computations in Section 2 of $t=|x|^{2}$ with 
$|U|^{2}$, replacing $x$ with $U$ and $\bar{x}$ with $U^{\star}$, we can easily obtain the following results comparing to Lemma 2.7, Lemma 2.8 and Lemma 2.9 .

$$
\begin{aligned}
& F_{A}^{+}=-\frac{1}{2}\left(f^{\prime}+f^{2}\right) U^{\star} d U \wedge d U^{\star} U, \\
& F_{A}^{-}=\left(\frac{1}{2} t f^{\prime}-\frac{1}{2} t f^{2}+f\right) d U^{\star} \wedge d U \\
& (\phi \wedge \phi)^{+}=-\frac{1}{2} g^{2} U^{\star} d U \wedge d U^{\star} U \\
& (\phi \wedge \phi)^{-}=-\frac{1}{2} g^{2} t d U^{\star} \wedge d U \\
& \left(d_{A} \phi\right)^{+}=-\frac{1}{2}\left(g^{\prime}+2 f g\right) U^{\star} d U \wedge d U^{\star} U \\
& \left(d_{A} \phi\right)^{-}=\left(\frac{1}{2} g^{\prime} t+g-f g t\right) d U^{\star} \wedge d U .
\end{aligned}
$$

The derivative here is taking the derivative of $|U|^{2}$.

Therefore, by (44), we could get an ODEs comparing to (6):

$$
\left\{\begin{array}{c}
f\left(|U|^{2}\right)^{\prime}+\lambda g\left(|U|^{2}\right)^{\prime}+f\left(|U|^{2}\right)^{2}-g\left(|U|^{2}\right)^{2}+2 \lambda f\left(|U|^{2}\right) g\left(|U|^{2}\right)=0 \\
t f\left(|U|^{2}\right)^{\prime}-t \lambda^{-1} g\left(|U|^{2}\right)^{\prime}+2 f\left(|U|^{2}\right)-2 \lambda^{-1} g\left(|U|^{2}\right)+g\left(|U|^{2}\right)^{2} t \\
-f\left(|U|^{2}\right)^{2} t+2 t f\left(|U|^{2}\right) g\left(|U|^{2}\right) \lambda^{-1}=0
\end{array}\right.
$$

The derivative here is the derivative of $|U|^{2}$.

Comparing this with (7), we are exactly solving the same equations. Therefore, comparing to Proposition 5.1, our construction gives solutions to the Kapsutin-Witten equations.

Proof of Theorem 1.3. By our construction, we have the freedom to choose $k$ real numbers $\lambda_{1}, \ldots, \lambda_{k}$ and $k$ quaternions $b_{1}, \ldots, b_{k}$ in $\mathbb{H}$ in Proposition 5.5. Therefore, we have a $5 k$ dimension family of solutions to the KapustinWitten equations.

\section{Nahm pole boundary solution over $S^{3} \times(0,+\infty)$}

In this section, we will show that our solutions in Section 4 can provide solutions on $S^{3} \times(0,+\infty)$ with Nahm pole boundary. 


\subsection{Nahm pole boundary condition}

Now, we will discuss what is a Nahm pole boundary condition to the Kapustin-Witten equations. Give a closed 3 -manifold $Y^{3}$, let $P$ to be a principle $S U(2)$ bundle over $Y^{3} \times(0,+\infty)$ and let $\operatorname{ad}(P)$ denote the adjoint bundle of $P$. Give a point $x \in Y^{3}$, for integer a=1,2,3, let $e_{a}$ be any orthonormal basis of $T_{x} Y$ and $t_{a} \in a d(P)$ satisfy the lie algebra relationship $\left[t_{a}, t_{b}\right]=\epsilon_{a b c} t_{c}$.

From [7] [13, we have the following definition of Nahm pole boundary condition on $Y^{3} \times(0,+\infty)$ and denote $y$ as the coordinate on $(0,+\infty)$.

Definition 6.1. A solution $(A, \phi)$ to $K W$ equation 2 over $Y^{3} \times(0,+\infty)$ satisfies the Nahm pole boundary if there exist orthonormal basis $e_{a}$ such that the Taylor expansion in y coordinate nears $y=0$ will be $\phi \sim \frac{\sum_{a=1}^{3} t_{a} e_{a}^{\star}}{y}+$ $\phi_{0}+\cdots, A \sim A_{0}+y a_{1}+\cdots$

\subsection{Nahm pole boundary condition over $S^{3} \times(0,+\infty)$}

In this subsection, we will describe the Nahm pole boundary condition on $S^{3}$ and show that our solution satisfy the Nahm pole boundary condition.

Now, we first describe the tangent space of $S^{3}$. Consider $S^{3}$ as the unit quaternion, $S^{3}=\left\{x=x_{1}+x_{2} I+x_{3} J+x_{4} K \in \mathbb{H} \mid x_{1}^{2}+x_{2}^{2}+x_{3}^{2}+x_{4}^{2}=\right.$ $1\}$, and the metric is induced by Euclidean metric on $\mathbb{R}^{4}$. Fix a point $x \in S^{3}$, we can identify the tangent space with vectors on $\mathbb{R}^{4}, T_{x} S^{3}=\left\{v \in \mathbb{R}^{4} \mid<\right.$ $v, x>=0\}$, here we consider $\mathrm{x}$ as a vector space on $\mathbb{R}^{4}$.

Define three orthnonormal basis

$$
\begin{aligned}
& e_{1}=\left(-x_{2}, x_{1},-x_{4}, x_{3}\right) \\
& e_{2}=\left(-x_{3}, x_{4}, x_{1},-x_{2}\right) \\
& e_{3}=\left(-x_{4},-x_{3}, x_{2}, x_{1}\right) .
\end{aligned}
$$

Obviously, we have $T_{x} S^{3}=\operatorname{span}\left\{e_{1}, e_{2}, e_{3}\right\}$.

So by the induced metric from the Euclidean metric on $\mathbb{R}^{4}$, we have the dual unit basis

$$
\begin{aligned}
& e_{1}^{\star}=-x_{2} d x_{1}+x_{1} d x_{2}-x_{4} d x_{3}+x_{3} d x_{4} \\
& e_{2}^{\star}=-x_{3} d x_{1}+x_{4} d x_{2}+x_{1} d x_{3}-x_{2} d x_{4} \\
& e_{3}^{\star}=-x_{4} d x_{1}-x_{3} d x_{2}+x_{2} d x_{3}+x_{1} d x_{4} .
\end{aligned}
$$


In addition, we have

$$
\begin{aligned}
\operatorname{Im}(\bar{x} d x)= & \left(-x_{2} d x_{1}+x_{1} d x_{2}-x_{4} d x_{3}+x_{3} d x_{4}\right) I \\
& +\left(-x_{3} d x_{1}+x_{4} d x_{2}+x_{1} d x_{3}-x_{2} d x_{4}\right) J \\
& +\left(-x_{4} d x_{1}-x_{3} d x_{2}+x_{2} d x_{3}+x_{1} d x_{4}\right) K \\
= & e_{1}^{\star} I+e_{2}^{\star} J+e_{3}^{\star} K .
\end{aligned}
$$

Therefore, $\left(0, \frac{\operatorname{Im}(\bar{x} d x)}{2}\right)$ can be consider as a leading term of Nahm pole boundary condition on $S^{3}$.

Now, we will show that the following singular solutions to the KapustinWitten equations over $\mathbb{R}^{4}$ can be consider as solutions to the KapustinWitten equations over $(0,+\infty) \times S^{3}$.

As the first equation of (2) are conformal invariant, consider the solutions

$$
\left\{\begin{array}{l}
A(x)=\operatorname{Im}\left(\frac{3 C}{C^{2}|x|^{4}+4 C|x|^{2}+1} \bar{x} d x\right) \\
\phi(x)=\operatorname{Im}\left(\frac{3 C\left(C|x|^{2}+1\right)}{\left(C^{2}|x|^{4}+4 C|x|^{2}+1\right)\left(C|x|^{2}-1\right)} \bar{x} d x\right) .
\end{array}\right.
$$

Using the following conformal transformation

$$
\begin{aligned}
\Psi:(0,+\infty) \times S^{3} & \rightarrow \mathbb{R}_{|x| \geq \frac{1}{\sqrt{C}}}^{4} \\
(y, \omega) & \rightarrow \frac{1}{\sqrt{C}} e^{y} \omega
\end{aligned}
$$

Then the pull back of 49 using $\Psi$ gives the following solution on $(0,+\infty) \times S^{3}$ :

$$
\left\{\begin{array}{l}
A(x)=\frac{6}{e^{4 y}+4 e^{2 y}+1} \sum_{a=1}^{3} t_{a} e_{a}^{\star} \\
\phi(x)=\frac{6\left(e^{2 y}+1\right)}{\left(e^{4 y}+4 e^{2 y}+1\right)\left(e^{2 y}-1\right)} \sum_{a=1}^{3} t_{a} e_{a}^{\star} .
\end{array}\right.
$$

It is easy to see that when $y \rightarrow 0, \frac{6\left(e^{2 y}+1\right)}{\left(e^{4 y}+4 e^{2 y}+1\right)\left(e^{2 y}-1\right)} \sim \frac{1}{y}$ and $y \rightarrow+\infty$, the solution exponentially decays. 
From Section 4, we get another solution to the Kapustin-witten equations (2),

$$
\left\{\begin{array}{l}
A(x)=\operatorname{Im}\left(\frac{1}{|x|^{2}} \frac{C^{2}|x|^{4}+C|x|^{2}+1}{C^{2}|x|^{4}+4 C|x|^{2}+1} \bar{x} d x\right) \\
\phi(x)=\operatorname{Im}\left(\frac{3 C\left(C|x|^{2}+1\right)}{\left(C^{2}|x|^{4}+4 C|x|^{2}+1\right)\left(C|x|^{2}-1\right)} \bar{x} d x\right)
\end{array}\right.
$$

Following the same process, we get another solution to the Kapustin-Witten equations with Nahm pole and exponentially decays.

Then the pull back of (51) using $\Psi$ gives the following solution on $(0,+\infty) \times S^{3}$ :

$$
\left\{\begin{array}{l}
A(x)=\frac{2}{e^{2 y}} \frac{e^{4 y}+e^{2 y}+1}{e^{4 y}+4 e^{2 y}+1} \sum_{a=1}^{3} t_{a} e_{a}^{\star} \\
\phi(x)=\frac{6\left(e^{2 y}+1\right)}{\left(e^{4 y}+4 e^{2 y}+1\right)\left(e^{2 y}-1\right)} \sum_{a=1}^{3} t_{a} e_{a}^{\star}
\end{array}\right.
$$

Therefore, we have the following theorem:

\section{Theorem 6.2.}

$$
\left\{\begin{array}{l}
A(x)=\frac{6}{e^{4 y}+4 e^{2 y}+1} \sum_{a=1}^{3} t_{a} e_{a}^{\star} \\
\phi(x)=\frac{6\left(e^{2 y}+1\right)}{\left(e^{4 y}+4 e^{2 y}+1\right)\left(e^{2 y}-1\right)} \sum_{a=1}^{3} t_{a} e_{a}^{\star}
\end{array}\right.
$$

is a solution to the Kapustin-Witten equations over $(0,+\infty) \times S^{3}$ with Nahm pole boundary with instanton number $+\frac{1}{2}$.

$$
\left\{\begin{array}{l}
A(x)=\frac{2}{e^{2 y}} \frac{e^{4 y}+e^{2 y}+1}{e^{4 y}+4 e^{2 y}+1} \sum_{a=1}^{3} t_{a} e_{a}^{\star} \\
\phi(x)=\frac{6\left(e^{2 y}+1\right)}{\left(e^{4 y}+4 e^{2 y}+1\right)\left(e^{2 y}-1\right)} \sum_{a=1}^{3} t_{a} e_{a}^{\star}
\end{array}\right.
$$

is a solution to the Kapustin-Witten equations over $(0,+\infty) \times S^{3}$ with Nahm pole boundary with instanton number $-\frac{1}{2}$. 
Proof. The computation of instanton number directly follows from Proposition 4.3.

\section{Acknowledgement}

The author greatly thanks Peter Burton, Anton Kapustin, Edward Witten, Jianfeng Lin, Ciprian Manolescu, Rafe Mazzeo and Yi Ni for their kindness and helpful discussions.

\section{References}

[1] M. F. Atiyah, Geometry of Yang-Mills fields, in: Mathematical problems in theoretical physics (Proc. Internat. Conf., Univ. Rome, Rome, 1977), Vol. 80 of Lecture Notes in Phys., 216-221, Springer, Berlin-New York (1978).

[2] M. F. Atiyah, N. J. Hitchin, V. G. Drinfeld, and Y. I. Manin, Construction of instantons, Phys. Lett. A 65 (1978), no. 3, 185-187.

[3] E. Corrigan and D. B. Fairlie, Scalar field theory and exact solutions to a classical SU(2) gauge theory, Phys. Lett. B 67 (1977), no. 1, 69-71.

[4] S. K. Donaldson and P. B. Kronheimer, The Geometry of FourManifolds, Oxford Mathematical Monographs, The Clarendon Press, Oxford University Press, New York (1990), ISBN 0-19-853553-8. Oxford Science Publications.

[5] D. Gaiotto and E. Witten, Knot invariants from four-dimensional gauge theory, Adv. Theor. Math. Phys. 16 (2012), no. 3, 935-1086.

[6] A. Kapustin and E. Witten, Electric-magnetic duality and the geometric Langlands program, Commun. Number Theory Phys. 1 (2007), no. 1, 1-236.

[7] R. Mazzeo and E. Witten, The Nahm pole boundary condition, in: The influence of Solomon Lefschetz in geometry and topology, Vol. 621 of Contemp. Math., 171-226, Amer. Math. Soc., Providence, RI (2014).

[8] W. Rudin, Functional Analysis, International Series in Pure and Applied Mathematics, McGraw-Hill, Inc., New York, second edition (1991), ISBN 0-07-054236-8.

[9] C. H. Taubes, Compactness theorems for $\mathrm{SL}(2 ; \mathbb{C})$ generalizations of the 4-dimensional anti-self dual equations, preprint (2013), arXiv: 1307.6447. 
[10] C. H. Taubes, PSL $(2 ; \mathbb{C})$ connections on 3 -manifolds with $\mathrm{L}^{2}$ bounds on curvature, Camb. J. Math. 1 (2013), no. 2, 239-397.

[11] K. K. Uhlenbeck, Connections with $L^{p}$ bounds on curvature, Comm. Math. Phys. 83 (1982), no. 1, 31-42.

[12] K. K. Uhlenbeck, Removable singularities in Yang-Mills fields, Comm. Math. Phys. 83 (1982), no. 1, 11-29.

[13] E. Witten, Fivebranes and knots, Quantum Topol. 3 (2012), no. 1, 1137.

Department of Mathematics, Caltech

1200 E California Blvd., Pasadena, CA 91125, USA

E-mail address: she@caltech.edu

ReCeIVED March 12, 2016 